\title{
Neuroprotective and cognitive enhancing effects of Clausena lansium (Lour.) skeels peels extract in ischemic rat brains
}

\author{
Wathita Phachonpai ${ }^{1 *}$, Terdthai Tongun ${ }^{2}$ \\ ${ }^{1}$ Division of Physiology, School of Medical Sciences, University of Phayao, Phayao, Thailand, 56000. \\ ${ }^{2}$ Department of Physiology, Faculty of Medicine, Khon Kaen University, Khon Kaen, Thailand, 40002.
}

\begin{tabular}{l}
\hline ARTICLE INFO \\
\hline Received on: $30 / 12 / 2020$ \\
Accepted on: $15 / 03 / 2021$ \\
Available online: 05/09/2021 \\
\hline Key words: \\
Clausena lansium (Lour.) \\
skeels, wampee peel, \\
oxidative stress, ischemic \\
stroke, memory impairment.
\end{tabular}

\begin{abstract}
Oxidative stress is a pivotal factor in the pathogenesis of many neurological disorders including ischemic stroke. Several scientific studies reveal that the peel fractions of some fruits are a source of bioactive compounds and exhibit stronger antioxidant activities. Here, we have elucidated the neuroprotective and cognitive enhancing effects of Clausena lansium (Lour.) Skeels or wampee (WP) peel crude extract administration on neuronal damage and memory deficit in rats exposed to focal cerebral ischemia. The antioxidant potential of WP peel extract was investigated in vitro. Furthermore, male Wistar rats received orally WP peel extract $(200,400$, and $600 \mathrm{mg} / \mathrm{kg}$ ) for a period of 7 days before and 21 days following permanent right middle cerebral artery occlusion (Rt. MCAO). WP peel extract possessed strong antioxidant activities in scavenging free radicals. However, in in vivo analysis, only the dose of $400 \mathrm{mg} / \mathrm{kg}$ showed significantly attenuated brain infarction and oxidation indices, restored memory and antioxidant enzyme activity, and improved the densities of survival neurons and cholinergic neurons in the hippocampal region compared to the MCAO-treated rats (all $p<0.05$ ). Our results provide new information about the cognitive enhancement and neuroprotective effects of WP peel extract. This suggests that WP peel extract may prove to be useful as an adjunct to ischemic stroke treatment.
\end{abstract}

\section{INTRODUCTION}

Ischemic stroke is one of the major causes of death and disability (Benjamin et al., 2017) and results from the occlusion of blood flow in a major brain artery, which initiates brain infarction and subsequent damage to neuronal cells that result in memory distortion (Román, 2013). Oxidative stress is considered to play a key mediator of ischemic brain injury (Kishimoto et al., 2019). It has been reported that free radicals are generated in the brain during ischemia, execute structural and functional alterations in the neurons, and may contribute to neuronal necrosis, which can further lead to cognitive function deficit (Rostamian et al., 2014). Up to now, there is no treatment available to cure poststroke dementia. The gold standard treatment for ischemic stroke focuses on preventing further

"Corresponding Author

Wathita Phachonpai, Division of Physiology, School of

Medical Sciences, University of Phayao, Phayao, Thailand, 56000.

E-mail: phachonpai@yahoo.com neuronal damage by maintaining constant cerebral blood flow and inhibiting the reactive oxygen species (ROS) generation with antioxidant agents (Kunz and Iadecola, 2009). Several studies have shown that most of the plants, including fruits, vegetables, and herbs, contain high levels of antioxidant compounds, and they could act as neuroprotective agents for cerebral ischemia (Chun et al., 2008).

Wampee (WP) or Clausena lansium (Lour.) Skeels is the scientific name and belongs to the family Rutaceae that is a native plant commonly found in Southern China. Every parts of WP, such as its leaves, peels, fruits, and seeds, is reported to have a high potential of several biological activities; for example, antimicrobial, anti-inflammatory, antitumor, hepatoprotective, antifungal, antiobesity, neuroprotective, and antioxidant effects. (Liu et al., 1996, 2019; Shen et al., 1989). In China and Thai traditional medicine, the fruit of WP is considered to be a cool fruit which has the effects to treat and prevent common cold, cough, dermatological problems, and malaria (Adebajo et al., 2009). In addition, this fruit shows an antispasmodic effect that relaxes the smooth muscles in the digestive system and relieves abdominal pain (Mahattanatawee et al., 2006). 
Up to date, with current research findings, the peel portions of some fruits have exhibited stronger antioxidant activity than the pulp portions (Elfalleh et al., 2012; Li et al., 2006). The previous study has shown that the WP peel exerts the antioxidant and anticancer activities in in vitro models: SGC-7901 human gastric carcinoma, HepG-2 human liver cancer cells, and A-549 human lung adenocarcinoma (Prasad et al., 2009a). Therefore, WP peel has the potential to be developed as a natural antioxidant supplementation. However, there is insufficient scientific evidence to substantiate that WP peel indeed has a neuroprotective effect even though it exhibits antioxidant and anticancer properties. Thus, this study presented herein investigates for the first time the usefulness of WP peel extract supplementation against memory impairment, brain infarct volume, densities of cholinergic and survival neurons, and oxidative stress indices after occlusion of the right middle cerebral artery occlusion (Rt. MCAO) in the rat model mimics the human ischemic stroke (Carmichael, 2005).

\section{MATERIALS AND METHODS}

\section{Preparation of the WP peel extract}

The ripe fresh fruits of WP were collected from Nan Province, Thailand, and immediately cleaned with tap water twice. WP peel can be left on the fruit, shed dried in the hot air oven at $40^{\circ} \mathrm{C}$, and triturated in order to create a fine powder. The ethanol maceration method was mixed with distilled water $(200 \mathrm{ml})$ and ethanol $(200 \mathrm{ml})$ for 24 hours and then filtered and evaporated by a rotary evaporator to yield a crude extract. The final yield of the aqueous extract used for this study was $10.93 \%$ and kept at $4{ }^{\circ} \mathrm{C}$ until further analysis and study in the animals.

\section{Antioxidant activity of WP peel extract determination}

The antioxidant activity of the WP peel extract was determined by the DPPH method of Kordali et al. (2005). Sample stock solutions of the extract were diluted at various concentrations of $20,40,60,80$, and $100 \mu \mathrm{g} / \mathrm{ml}$ in methanol. $2 \mathrm{ml}$ of DPPH methanol solution was added to sample solutions. The reaction mixture was shaken immediately in the dark for 20 minutes until the reaction plateau was reached in the systems. Absorbance was read at $517 \mathrm{~nm}$ and calculated as follows:

DPPH scavenging

(\%) or Percent inhibition $=A_{\text {control }}-A_{\text {sample }} / A_{\text {control }} \times 100$

where $A_{\text {control }}$ and $A_{\text {sample }}$ are the absorbances of the control and the standard sample of the WP peel extract.

\section{Animals and experimental design}

The following studies were permitted by the Animal Ethics Committee of the University of Phayao, Thailand (No. 5801040005). All experimental protocols and surgical techniques were carried out according to the Principles of Laboratory Animal Care (National Institutes of Health publication, \#85-23, revised in 1985). Forty-eight adult male Wistar rats (200-250 g) from the National Laboratory Animal Center, Salaya, Nakorn Pathom, Thailand, were used in this study. All rats were housed at an ambient temperature of $22^{\circ} \mathrm{C} \pm 2{ }^{\circ} \mathrm{C}$ with an artificial light-dark cycle (12 hours) and allowed free access to a commercial pellet diet and water.
The animals were divided into six groups ( $n=8$ /group): sham control-treated group; MCAO control group, all rats in this group which received distill water (vehicle $1 \mathrm{cc}$ ); donepezil (1 mg/ $\mathrm{kg}$ ) + MCAO group (positive control); and three treatment groups as follows: WP peel extract $(200 \mathrm{mg} / \mathrm{kg})+\mathrm{MCAO}$, WP peel extract $(400 \mathrm{mg} / \mathrm{kg})+\mathrm{MCAO}$, and WP peel extract $(600 \mathrm{mg} / \mathrm{kg})$ + MCAO. All doses of this extract were chosen on the basis of a pilot study conducted in our laboratory (Tongun and Phachonpai, 2020).

All rats were administered with the assigned substances via the feeding tube once daily continuously for 7 days before and 21 days after the MCAO surgery. At the end of the experimental period, cognitive performances were determined using the Morris water maze (for spatial memory) and the passive avoidance test (for nonspatial memory). Rats were sacrificed on the 29th day to determine the brain infarct volume, densities of survival and cholinergic neurons, and oxidative markers such as glutathione peroxidase (GPx) activity and lipid peroxidation malondialdehyde (MDA) level.

\section{Focal cerebral ischemia induction}

Anesthesia was induced in rats with thiopental sodium $(50 \mathrm{mg} / \mathrm{kg})$ intraperitoneal (i.p.) injection for Rt. MCAO surgery as described by Longa et al. (1989). Surgery was conducted with an aseptic technique. The internal carotid and the right external carotid arteries were exposed through a neck midline incision. A 4-0 nylon monofilament suture coated with silicone was inserted into the common carotid artery and pushed through the internal carotid artery $(19-21 \mathrm{~mm})$ from the common carotid artery bifurcation. Sham-operated animals were subjected to the same surgical method, but the filament is not inserted into the middle cerebral artery. The wound was closed and applied with $10 \%$ povidine solution for antiseptic postoperative treatment.

\section{Morris water maze test}

This test was adapted from the method of Morris (1994). A circular pool $(170 \times 60 \mathrm{~cm})$ was filled with water $\left(22^{\circ} \mathrm{C} \pm 2{ }^{\circ} \mathrm{C}\right.$, depth $40 \mathrm{~cm}$ ) and made opaque with milk powder. The pool was separated into four quadrants. An escape platform $(10 \mathrm{~cm}$ in diameter) was hidden below the powder water surface $(2 \mathrm{~cm})$ and was located in the same location of the pool. The time to reach the platform is called escape latency. In order to determine the retention memory, it was measured on the next day. In this case, the platform was brought out of the water maze and the rat was allowed to swim for 60 seconds. The time spent to search the platform in its previous location in the target quadrant was reported in terms of the retention time which indicates the level of memory consolidation.

\section{Passive avoidance test}

In this case, rats learned to avoid a dangerous environment such as a foot shock. The equipment consisted of a two-compartment box $(50 \times 50 \times 35 \mathrm{~cm})$ and light and dark compartments, with a sliding door between the two. The floor of the dark compartment was created out of stainless steel rods through which the foot shock was delivered from a constant current Lafayette Master Shocker (Model 2400SS). The rat was allowed to survey both of the compartments for 5 minutes, 3 test trials per day. On the day of the test trial, as 
soon as the rat had walked into the dark compartment, the sliding door between the two compartments was closed and a single foot shock was transported through the steel rods's floor $(50 \mathrm{~Hz}, 1 \mathrm{~mA}$, for 1 seconds). The rat was left in the dark compartment for further about 20 seconds to permit the animal to form a link between the properties of the compartment and the foot shock. In order to determine the retention memory, the rat was positioned in the bright compartment and time taken to enter the bright compartment for the time was recorded using a stopwatch. Normal rats evaded entering the compartment where they had previously been exposed to the foot shock. Decreased time spent in the bright compartment recommended poor memory retention.

\section{Determination of cerebral infarct volume}

The determination of cerebral infarct size was estimated by the triphenyl tetrazolium chloride (TTC) staining method. The nonischemic area was stained red while the brain infarct area was not stained and presented as pale. Stained brain slices were digitized and the area of infarct size with a computerized image analysis system was analyzed (Optimas 6.1; Media Cybernetics, Silver Springs, MD).

\section{Nissl staining and survival neuron densities counts}

All adjacent serial coronal hippocampal tissues were mounted on slides, air-dried for 24 hours, hydrated in distilled water, stained with cresyl violet $(0.75 \%)$, dehydrated in the graded alcohols $(70 \%, 95 \%, 100 \%)$, cleared in xylene, cover-slipped, and examined under a light microscope model BH-2 (Olympus, 40× objective). The viewer was blinded throughout investigation. The neuronal survival cells were counted on the basis of a stained cell with at least two processes.

\section{Determination of the cholinergic neuron densities}

The $30 \mu \mathrm{m}$ thickness of hippocampal area sections was cut by a cryostat and was stained for choline acetyltransferase expression with the avidin-biotin-peroxidase method. 0.01 $\mathrm{M}$ phosphate-buffered saline was used to dilute the reagents and washing of brain tissue sections in each of the steps. All hippocampal tissue sections were stained for 120 minutes with biotinylated goat anti-rabbit IgG secondary antibody and 3,3'-diaminobenzidine (Sigma-Aldrich Chemical Co., St. Louis, MO) and $0.01 \% \mathrm{H}_{2} \mathrm{O}_{2}$. Finally, the brain-stained sections were mounted on the slides, washed in water, cleared with xylene, and cover-slipped.

\section{Measurement of lipid peroxidation}

Brain MDA levels were determined by using the thiobarbituric acid method described by Okhawa et al. (1979). Briefly, the cortex tissues were homogenized with 10 times $(w / v)$ ice-cold 0.1 sodium phosphate buffer $(\mathrm{pH} 7.4)$. The reagents of

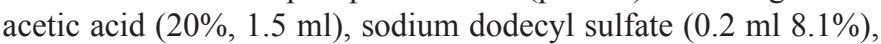
and thiobarbituric acid $(1.5 \mathrm{ml} 0.8 \%)$ were added to $0.1 \mathrm{ml}$ brain tissue sample. The mixture solution was heated at $100^{\circ} \mathrm{C}$ for 60 minutes in a boiling water bath and cooled in tap water bath for 10 minutes. After cooling, the mixture of distilled water $(1.0 \mathrm{ml})$ and $\mathrm{n}$-butanol and pyridine $(5 \mathrm{ml})$ was added and centrifuged $(4,000$ $\mathrm{rpm}$ ) for 10 minutes. The absorbance was detected at $532 \mathrm{~nm}$ to detect MDA concentrations.

\section{Determination of GPx activity}

GPx activity was measured according to the method described by Rotruck et al. (1973). Briefly, the mixture contained phosphate buffer (0.1 M, pH 7.4), sodium azide (10 Mm), GSH (4 $\mathrm{mM}), \mathrm{H} 2 \mathrm{O} 2(2.5 \mathrm{mM})$, and $0.5 \mathrm{ml}$ of brain supernatant and was mixed gently by vortex, then incubated at $37^{\circ} \mathrm{C}$ for 10 minutes, and added to $0.4 \mathrm{ml}$ of $10 \% \mathrm{TCA}$, and the whole was centrifuged at 3,000 rpm for 5 minutes. Determination of glutathione was carried out using enzymatic cycling and Ellman's reagent $\left(5,5^{\prime}\right.$-dithiobis-2-nitrobenzoic acid) in $0.1 \%$ sodium nitrate, and the absorbance was detected at $412 \mathrm{~nm}$ against a blank.

\section{Statistical analysis}

Data were expressed as mean \pm SEM. Statistical differences among the experimental groups were scrutinized using one-way analysis of variance and succeeded by post hoc Tukey's test; $p<0.05$ was considered to be statistically significant.

\section{RESULTS}

\section{Effect of WP peel extract on scavenging ability}

WP peel extract exhibited high free radical scavenging activity against DPPH-free radical, with $\mathrm{IC}_{50}$ value of $60 \mu \mathrm{g} / \mathrm{ml}$, comparable to that of ascorbic acid, a standard agent (Table 1).

\section{Effect of WP peel extract on memory impairment}

The rats in the MCAO group exhibited memory deficit in both escape latency and retention time as compared to shamoperated control $(p<0.05)$ (Fig. 1). In addition, in passive avoidance testing (Fig. 2), the latency time to enter the bright compartment was significantly shorter in the rats with permanent $\mathrm{MCAO}$, as compared to the sham-operated control $(p<0.05)$. Based on these results, it is clear that the cognitive impairment was significantly established in the MCAO-treated group. On the contrary, only the WP peel extract $(400 \mathrm{mg} / \mathrm{kg}$ ) and donepeziltreated groups significantly decrease in the time of escape latency but increase the retention time as compared to the MCAO-treated group $(p<0.05)$. Moreover, rats exposed to this extract at a dose of $400 \mathrm{mg} / \mathrm{kg}$ demonstrated inducing the time spent in the bright compartment as compared to the MCAO-treated group ( $p<$ 0.05 ), which suggested that the medium dose of WP peel extract treatment restored the learning and memory impairment induced by the MCAO method.

Unfortunately, no significant alteration in these parameters was observed in low dose and high dose of WP peel extract.

Table 1. $\mathrm{IC}_{50}$ values $(\mu \mathrm{g} / \mathrm{ml})$ of WP peel extract at different concentrations on the DPPH scavenging ability.

\begin{tabular}{cc}
\hline Concentrations $(\mu \mathrm{g} / \mathrm{ml})$ & IC $_{50}$ value $(\%)$ \\
\hline 20 & 25.60 \\
40 & 40.34 \\
60 & $59.75^{*}$ \\
80 & 65.80 \\
100 & 80.24 \\
\hline
\end{tabular}

Values reported are as means $\pm \mathrm{SD} . *$ significant at $p<0.05$. 


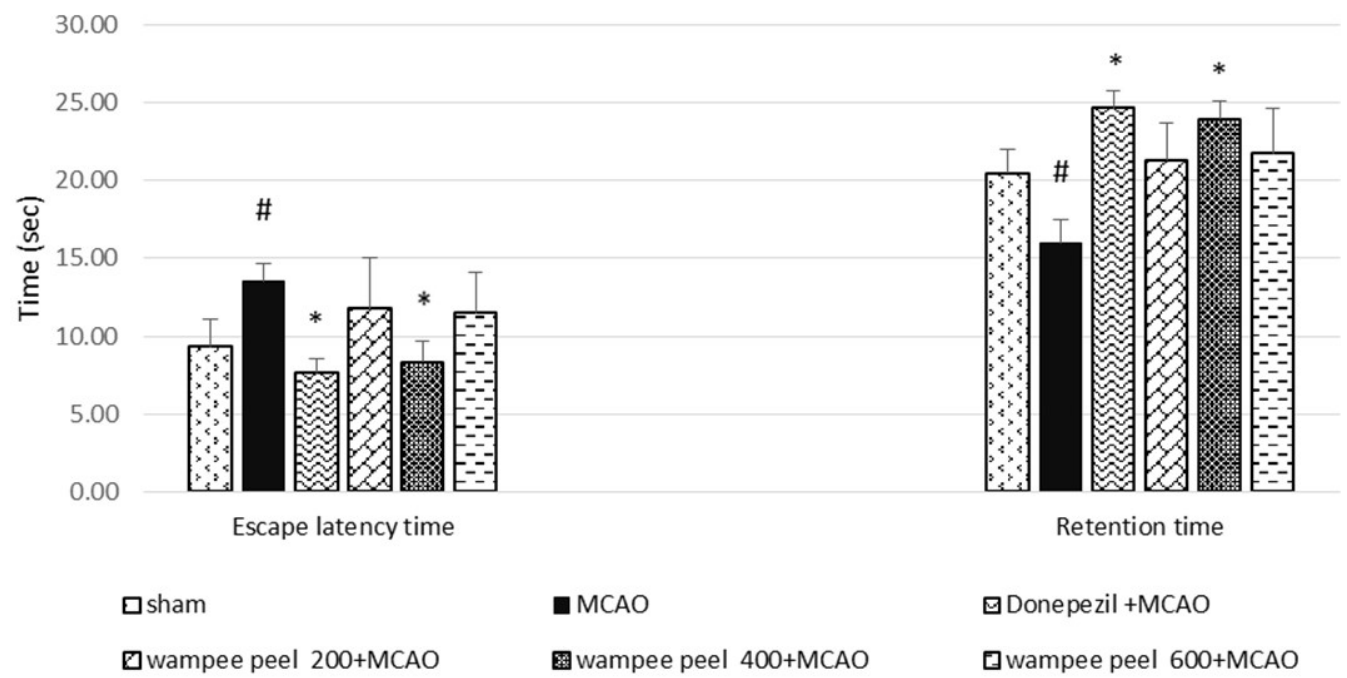

Figure 1. Effect of WP peel extract on the escape latency and retention time in the Morris water maze test. ${ }^{\prime} p<0.05$ versus sham control; ${ }^{*} p<0.05$ versus MCAO control.

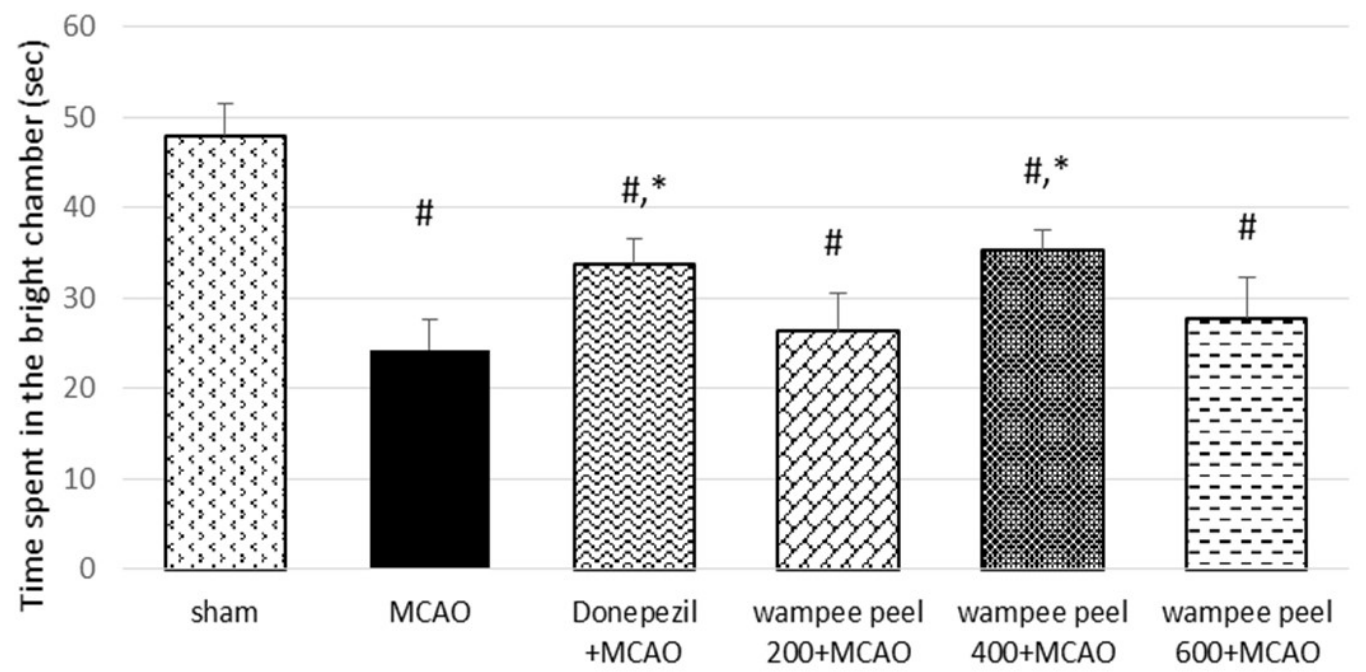

Figure 2. Effect of WP peel extract on the time spent in the bright compartment in the passive avoidance test. ${ }^{\#} p<0.05$ versus sham control; $" p<0.05$ versus MCAO control.

\section{Effect of WP extract on the volume of brain infarction}

TTC straining of rat brain sections in the MCAO group showed that the infarct area was significantly greater than that in the sham group $(p<0.05)$. However, WP peel extract $(400 \mathrm{mg} / \mathrm{kg})$ and donepezil reduced the brain infarct volume significantly $(p<$ 0.05) (Fig. 3).

\section{Effect of WP peel extract on the densities of survival neuron in hippocampus}

Nissl's staining of the various brain areas of the sectioned hippocampus obtained from MCAO-treated rats exhibited marked reductions of survival neuron densities compared to the shamtreated group $(p<0.05)$. Interestingly, a medium dose of this crude extract treatment was able to cause significant $(p<0.05)$ enhancement in the neuronal survival cells in the hippocampal region except in $\mathrm{CA} 2$ as compared to the MCAO-treated group (Fig. 4).

\section{Effect of WP peel extract on the densities of cholinergic neuron in hippocampus}

There was a decrease in the cholinergic neuron densities $(p<0.05)$ in all areas of hippocampus of MCAO-treated rats. Treatment with donepezil significantly elevated cholinergic cell densities in all regions of the hippocampus as compared to the MCAO-treated group $(p<0.05)$. Again, rats subjected to WP peel extract treatment at a medium dose significantly enhanced cholinergic neurons in all hippocampal regions, except CA2 fields $(p<0.05)$ when compared to the MCAO group (Fig. 5). 


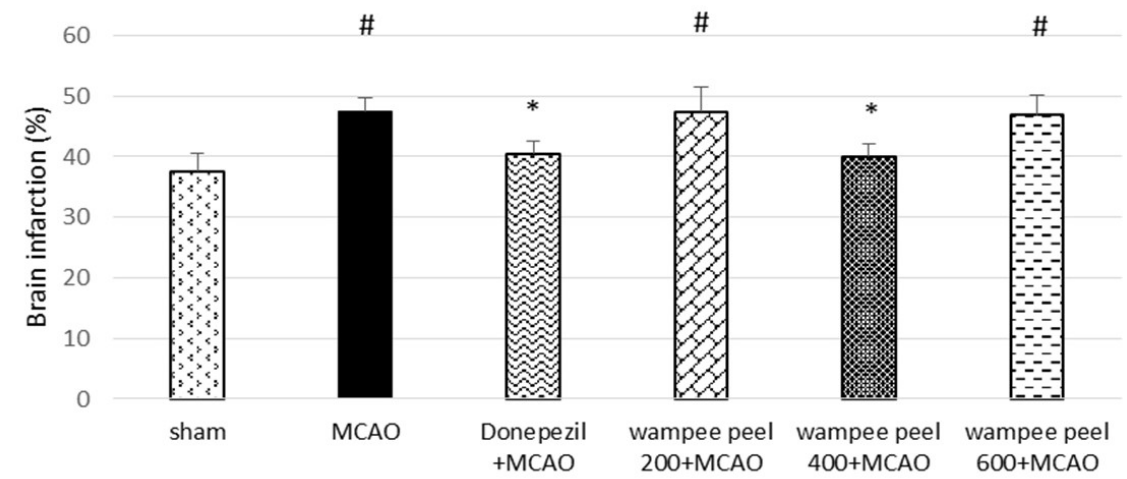

Figure 3. Effect of WP peel extract on the brain infarct size. ${ }^{\sharp} p<0.05$ versus sham control; ${ }^{*} p<0.05$ versus MCAO control.

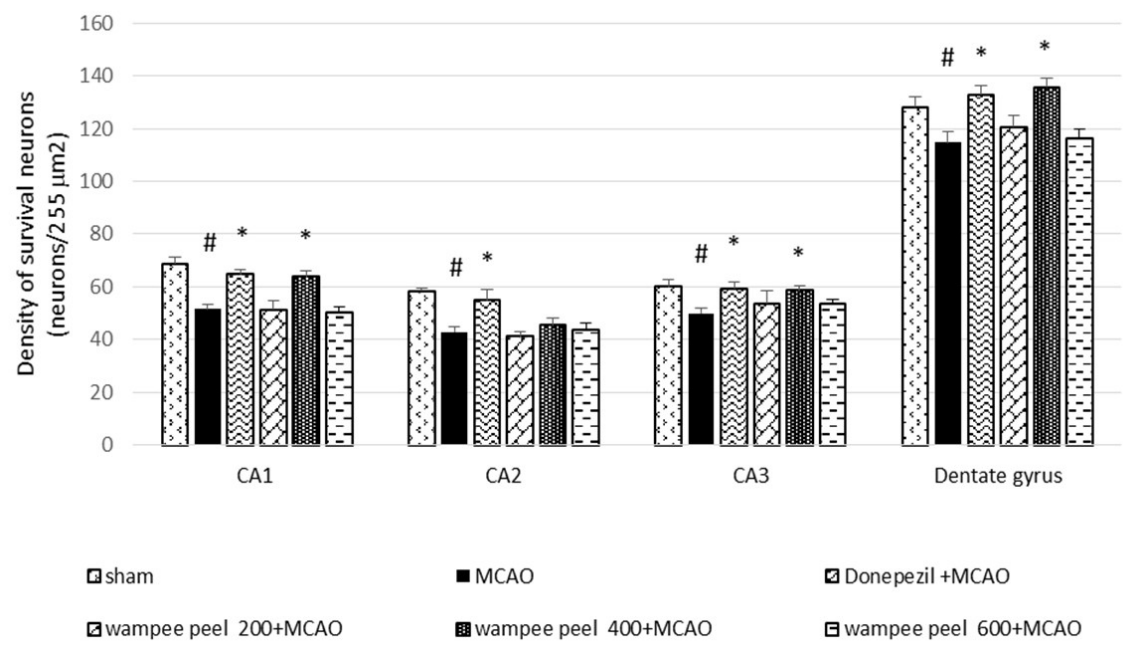

Figure 4. Effect of WP peel extract on the densities of survival neurons in hippocampal regions. ${ }^{\#} p<$ 0.05 versus sham control; " $p<0.05$ versus MCAO control.

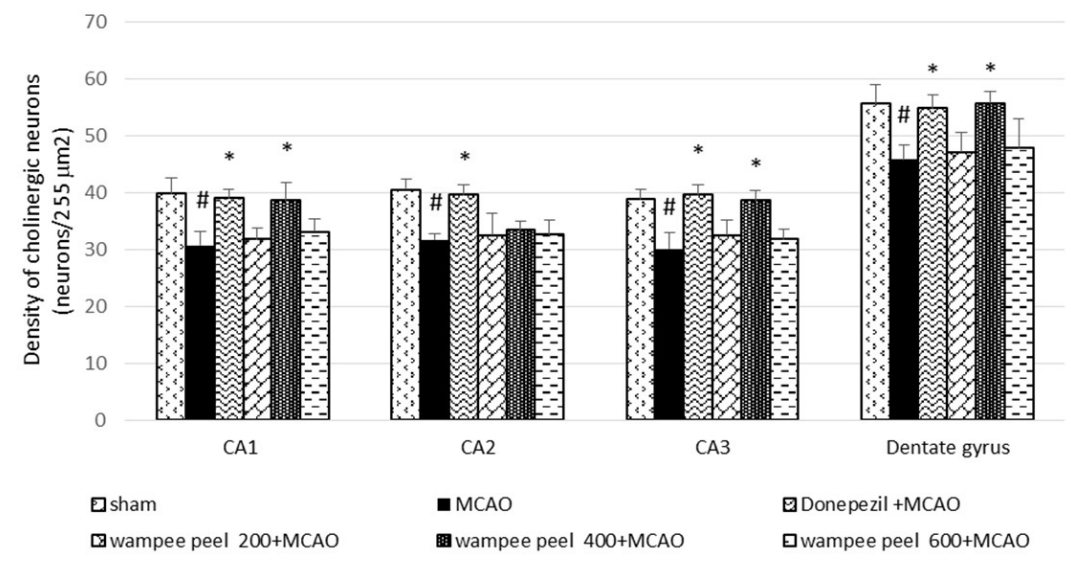

Figure 5. Effect of WP peel extract on the densities of cholinergic neurons in hippocampal regions. ${ }^{*} p<$ 0.05 versus sham control; ${ }^{*} p<0.05$ versus MCAO control. 
Table 2. Effect of WP peel extract on the oxidative stress biomarkers in rat brains.

\begin{tabular}{lcc}
\hline Treatment & MDA level (nmol/ml) & GPx activity (mU/mg protein) \\
\hline Sham control & $2.14 \pm 0.33$ & $15.16 \pm 0.67$ \\
MCAO control & $5.51 \pm 0.28^{*}$ & $12.86 \pm 1.71^{*}$ \\
MCAO + donepezil & $3.13 \pm 0.22^{* *}$ & $17.33 \pm 0.14^{* * * *}$ \\
MCAO + WP peel 200 & $5.12 \pm 0.54^{*}$ & $14.06 \pm 1.97$ \\
MCAO + WP peel 400 & $3.02 \pm 0.23^{* *}$ & $18.64 \pm 1.31^{*, * *}$ \\
MCAO + WP peel 600 & $5.07 \pm 0.46^{*}$ & $18.17 \pm 1.83^{*}$ \\
\hline
\end{tabular}

" $p<0.05$ versus sham control; " $p<0.05$ versus MCAO control.

\section{Effect of WP peel extract on MDA level and GPx activity}

A significant increase $(p<0.05)$ in MDA level was detected in the MCAO group as compared to sham animals. Rats of the WP peel extract $(400 \mathrm{mg} / \mathrm{kg})$ and donepezil-pretreated MCAO group exhibited significant attenuation $(p<0.05)$ in brain MDA level in comparison to MCAO group rats. Excess of free radicals is associated with ischemia in MCAO procedure-induced change in brain antioxidant enzyme. GPx activity significantly $(p<$ $0.05)$ decreased in the MCAO group when compared to the sham group $(p<0.05)$. However, WP peel extract $(400 \mathrm{mg} / \mathrm{kg})$ treatment reversed the change of this enzyme which indicated that the medium dose of WP peel extract demonstrated the ability to restore antioxidant activity on ischemic brain damage in the rat (Table 2).

\section{DISCUSSION}

The present study was undertaken to determine the potential effects of WP peel extract against ischemia following MCAO-treated rats on the improvement of oxidative stress status as well as cognitive behavioral and histopathological alterations.

MCAO is one of the widely used focal stroke models in the rodent (Yang et al., 2006). The advantages of the MCAO model are its good reproducibility of brain infarct size and neurological problems, which are similar in human stroke (Kaiser and West, 2020). A previous study has also reported that the MCAO procedure induces excessive free radicals leading to neuronal death, resulting in memory impairment in rats (Gao et al., 2017). Similarly, the present study demonstrated MCAO results in cortical infarction as well as damage to the hippocampal cholinergic neuronal damage. In addition, our results showed that oxidative stress is strongly associated with MCAO and we suggest that it may be a crucial role in the pathogenesis of the cerebral ischemic stroke. Depletion of GPx and antioxidant enzyme, and elevation of MDA in the brain were obvious in the MCAO-treated rats. Furthermore, our study revealed that memory impairment in the MCAO-exposed group in the Morris water maze test and the passive avoidance task were significantly correlated with an increase in oxidative stress, which induces neuronal cell death. These findings support the hypothesis that MCAO contributes to the interruption of blood flow and promotes ROS generation in the brain and leads to cerebral infarction, subsequently promoting the hippocampal neuronal cell and cholinergic neurons damage, and finally results in memory distortion.

WP fruits contain various bioactive compounds as therapeutic alternatives (Prasad et al., 2009b). However, up to date, no reports on the neuroprotective and cognitive enhancing effects of WP peel extract against ischemia-induced neuronal damage and cognitive decline. Herein, WP peel extract at a dosage of $400 \mathrm{mg} / \mathrm{kg}$ had a therapeutic cognitive improving effect in both passive avoidance and Morris water maze tests. All these effects of this extract were similar to that of the standard antidementia drug, donepezil. Additionally, we investigated the possible mechanisms of WP peel extract to improve cognitive functions in rat model of ischemic stroke. The results showed that a medium dose of WP peel extract significantly elevated GPx activity, decreased MDA level, reduced cerebral infarct volume, and protected against ischemic cholinergic and neuronal cell death in hippocampus. In addition, our data also showed that all fractions of this crude extract act as radical scavengers and are able to react with the DPPH-free radical. Therefore, the cognitive improvement induced by WP peel extract may occur partly via the antioxidant properties and the promoting cholinergic function. Our result is in conformity with another study which reported that the bioactivities of WP peel extract exhibited strong antioxidant activity (Deng et al., 2012). However, this is the first report that describes the neuroprotective and cognitive enhancing effects of WP peel extract supplementation in ischemic stroke.

Cumulative evidence reports that ischemia induced neurogenesis in areas of the adult brain such as striatum and subgranular zone (SGZ) (Arvidsson et al., 2002; Parent et al., 2002). Herein, our results found that there were higher survival neurons and cholinergic neuron densities in CA1, CA3, and dentate gyrus (DG) of rats group treated with WP peel extract at a dose of $400 \mathrm{mg} / \mathrm{kg}$. It has been reported that CA1 and CA3 play a critical role in encoding and retrieval memory processes, respectively (Jinzhao et al., 2008). The DG is an important plastic region in the mammalian brain; novel neuronal cells are generated and functionally integrated into mature circuits in hippocampus and contribute to learning and memory functions in particular. The present results were consistent with previous studies which identified the potential of plants and their active compounds promoting neurogenesis after stroke condition (Zhang et al., 2014). Thus, it is possible to infer that the medium dose of this crude extract favored the neurogenesis in SGZ of the DG regions. However, BrdU immunohistochemistry for studying the neurogenesis is needed to arrive at a final conclusion. Moreover, the role of WP peel in neuronal apoptosis still remains to be clarified.

Unfortunately, the low and high doses of WP peel extracts did not show any significant alteration in markers of oxidative stress and cognitive decline, suggesting that the WP peel extract used in this study was not the pure compound. There are various bioactive compounds in this extract; some pure compounds are active, but some of them are inactive, depending on compounds and the structural relationship between functional groups and the targeting site of the compounds react to it. Therefore, the antioxidant and the cognitive enhancing effect of WP peel extract may occur via the additive/noninteractive, antagonistic, or synergistic effects of several bioactive compounds in these crude extracts.

A limitation of this study is that all active ingredients of WP peel extract are not investigated. In addition, direct and interaction effects of various ingredients are still possible. Therefore, further studies are still required to provide a better understanding of the active ingredients of this extract. 
Taking all data together, WP peel extract has the ability to combat against memory deficit in focal cerebral ischemia by neutralizing the free radical, suppressing the accumulation of lipid peroxidation products, reducing the brain infarct volume, thus maintaining healthy cholinergic neurons and neuronal cells in the hippocampus.

\section{CONCLUSION}

The major findings of our study have suggested that WP peel extract might be a potential neuroprotective agent for ischemic stroke because it attenuates MCAO-induced cognitive discrepancies, improved cholinergic deficit, and decreased neurodegeneration in hippocampus. In addition, the reduced oxidative stress, the restored antioxidant enzyme, and the direct ability to scavenge free radical activity of WP peel may also contribute to the role. However, future studies should contribute to a further understanding of the identification of major bioactive ingredients responsible for its neuroprotective and cognitive enhancing effects.

\section{ACKNOWLEDGMENT}

The authors are grateful to Professor Dr. Jintanaporn Wattanathorn, Division of Physiology, Faculty of Medicine, Khon Kaen University, for her kind assistance to use the facilities of the laboratory.

\section{AUTHOR CONTRIBUTIONS}

All authors made substantial contributions to conception and design, acquisition of data, or analysis and interpretation of data; took part in drafting the article or revising it critically for important intellectual content; agreed to submit to the current journal; gave final approval of the version to be published; and agree to be accountable for all aspects of the work. All the authors are eligible to be an author as per the international committee of medical journal editors (ICMJE) requirements/guidelines.

\section{CONFLICT OF INTEREST}

All authors declare that they do not have any conflicts of interest related to this work.

\section{FUNDING}

This study was financially supported by the University of Phayao, Thailand, with Grant no. R020058217044.

\section{PUBLISHER'S NOTE}

This journal remains neutral with regard to jurisdictional claims in published institutional affiliation.

\section{REFERENCES}

Adebajo AC, Iwalewa EO, Obuotor EM, Ibikunle GF, Omisore NO, Adewunmi CO, Obaparusi OO, Klaes M, Adetogun GE, Schmidt TJ, Verspohl EJ. Pharmacological properties of the extract and some isolated compounds of Clausena lansium stem bark: anti-trichomonal, antidiabetic, anti-inflammatory, hepatoprotective and antioxidant effects. J Ethnopharmacol, 2009; 122:10-9.

Arvidsson A, Collin T, Kirik D, Kokaia Z, Lindvall O. Neuronal replacement from endogenous precursors in the adult brain after stroke. Nat Med, 2002; 8:963-70.

Benjamin EJ, Blaha MJ, Chiuve SE, Cushman M, Das SR, Deo R, de Ferranti SD, Floyd J, Fornage M, Gillespie C, Isasi CR, Jiménez
MC, Jordan LC, Judd SE, Lackland D, Lichtman JH, Lisabeth L, Liu S, Longenecker CT, Mackey RH, Matsushita K, Mozaffarian D, Mussolino ME, Nasir K, Neumar RW, Palaniappan L, Pandey DK, Thiagarajan RR, Reeves MJ, Ritchey M, Rodriguez CJ, Roth GA, Rosamond WD, Sasson C, Towfighi A, Tsao CW, Turner MB, Virani SS, Voeks JH, Willey JZ, Wilkins JT, Wu JH, Alger HM, Wong SS, Muntner P, American Heart Association Statistics Committee and Stroke Statistics Subcommittee. Heart Disease and Stroke Statistics-2017 Update: a report from the American Heart Association. Circulation, 2017; 135:e146-603.

Carmichael ST. Rodent models of focal stroke: size, mechanism, and purpose. NeuroRx, 2005;2:396-409.

Chun HS, Kim JM, Choi EH, Chang N. Neuroprotective effects of several korean medicinal plants traditionally used for stroke remedy. J Med Food, 2008;11:246-51.

Deng GF, Shen C, Xu XR, Kuang RD, Guo YJ, Zeng LS, Gao LL, Lin X, Xie JF, Xia EQ, Li S, Wu S, Chen F, Ling WH, Li HB. Potentia of fruit wastes as natural resources of bioactive compounds. Int J Mol Sci, $2012 ; 13: 8308-23$.

Elfalleh W, Hannachi H, Tlili N, Yahia Y, Nasri N, Ferchichi A. Total phenolic contents and antioxidant activities of pomegranate peel, seed, leaf and flower. J Med Plants Res, 2012; 6:4724-30.

Gao XJ, Xie GN, Liu L, Fu ZJ, Zhang ZW, Teng LZ. Sesamol attenuates oxidative stress, apoptosis and inflammation in focal cerebral ischemia/reperfusion injury. Exp Ther Med, 2017; 14:841-7.

Jinzhao J, Stephen M. Differential roles for hippocampal areas $\mathrm{CA} 1$ and $\mathrm{CA} 3$ in the contextual encoding and retrieval of extinguished fear. Learn Mem, 2008; 15:244-51.

Kaiser EE, West FD. Large animal ischemic stroke models: replicating human stroke pathophysiology. Neural Regen Res, 2020; 15:1377-87.

Kishimoto M, Suenaga J, Takase H, Araki K, Yao T, Fujimura T, Murayama K, Okumura K, Ueno R, Shimizu N, Kawahara N, Yamamoto T, Seko Y. Oxidative stress-responsive apoptosis inducing protein (ORAIP) plays a critical role in cerebral ischemia/reperfusion injury. Sci Rep, 2019; 9:13512.

Kordali S, Cakir A, Mavi A, Kilic H, Yildirim A. Screening of chemical composition and antifungal and antioxidant activities of the essential oils from three Turkish artemisia species. J Agric Food Chem, $2005 ; 53: 408-16$

Kunz A, Iadecola C. Cerebral vascular dysregulation in the ischemic brain. Handb Clin Neurol, 2009; 92:283-305.

Li Y, Guo C, Yang J, Wei J, Xu J, Cheng S. Evaluation of antioxidant properties of pomegranate peel extract in comparison with pomegranate pulp extract. Food Chem, 2006; 96:254-60.

Liu GT, Li WX, Chen YY, Wei HL. Hepatoprotective action of nine constituents isolated from the leaves of Clausena lansium in mice. Drug Dev Res, 1996; 39:174-8.

Liu YP, Guo JM, Liu YY, Hu S, Yan G, Qiang L, Fu YH Carbazole Alkaloids with potential neuroprotective activities from the fruits of Clausena lansium. J Agric Food Chem, 2019; 67:5764-71.

Longa EZ, Weinstein PR, Carlson S, Cummins R. Reversible middle cerebral artery occlusion without craniectomy in rats. Stroke, 1989, 20:84-91.

Mahattanatawee K, Manthey JA, Luzio G, Talcott ST, Goodner $\mathrm{K}$, Baldwin EA. Total antioxidant activity and fiber content of select Florida-grown tropical fruits. J Agric Food Chem, 2006; 54:7355-63.

Morris RG. Working memory in Alzheimer-type dementia. Neuropsychology, 1994; 8:544-54.

Okhawa H, Ohishi N, Yagi K. Assay of lipid peroxides in animals tissue by thiobarbituraic acid reaction. Anal Biochem, 1979; 95:351-8.

Parent JM, Vexler ZS, Gong C, Derugin N, Ferriero DM. Rat forebrain neurogenesis and striatal neuron replacement after focal stroke. Ann Neurol, 2002; 52:802-13.

Prasad KN, Hao J, Yi C, Zhang D, Qiu S, Jiang Y, Zhang M, Chen F. Antioxidant and anticancer activities of wampee [Clausena lansium (Lour.) Skeels] peel. J Biomed Biotechnol, 2009a; 2009:612805. 
Prasad NK, Yang B, Ruenroengklin N, Zhao M, Jiang Y. Application of ultrasonication or high pressure to assist extraction of flavonoids from litchi fruit pericarp. J Food Process Eng, 2009b; 32:828-43.

Román GC. Vascular dementia: distinguishing characteristics, treatment, and prevention. J Am Geriatr Soc, 2003; 51:S296-304.

Rostamian S, Mahinrad S, Stijnen T, Sabayan B, Craen AJ. Cognitive impairment and risk of stroke: a systematic review and metaanalysis of prospective cohort studies. Stroke, 2014; 45:1342-8.

Rotruck JT, Pope AL, Ganther HE, Swanson AB. Selenium: biochemical roles as a component of glutathione peroxidase. Science, 1973; 179:588-90.

Shen ZF, Chen QM, Liu HF, Xie MZ. The hypoglycemic e-ect of clausenacoumarine. Yao Xue Xue Bao, 1989; 24:391-2.

Tongun T, Phachonpai W. Cognitive booster of wampee peel extract on chronic restraint stress-induced memory dysfunction in rats. $\mathrm{J}$ Appl Pharm Sci, 2020; 10:019-26.
Yang SH, Shetty RA, Liu R, Sumien N, Heinrich KR, Rutledge M, Thangthaeng N, Brun-Zinkernagel AM, Forster MJ. Endovascular middle cerebral artery occlusion in rats as a model for studying vascular dementia. Age (Dordr), 2006; 28:297-307.

Zhang E, Shen J, So KF. Chinese traditional medicine and adult neurogenesis in the hippocampus. J Tradit Complement Med, 2014; 4: $77-81$.

\section{How to cite this article:}

Phachonpai W, Tongun T. Neuroprotective and cognitive enhancing effects of Clausena lansium (Lour.) skeels peels extract in ischemic rat brains. J Appl Pharm Sci, 2021; 11(09):001-008. 